\title{
Intra-vital Observation of Lung Water Retention Following Intravenous Injection of Anti-MHC-class I (H-2K) Monoclonal Antibody in Mice
}

\author{
HIDEYUKI OCHI ${ }^{1}$, TAKEHIKO IIJIMA ${ }^{1}$ and AKIRA USHIYAMA ${ }^{2}$ \\ ${ }^{1}$ Department of Perioperative Medicine, Division of Anesthesiology, \\ Showa University, School of Dentistry, Tokyo, Japan; \\ ${ }^{2}$ Department of Environmental Health, National Institute of Public Health, Saitama, Japan
}

\begin{abstract}
Background/Aim: Leukocyte activation is thought to be a major step in sepsis-induced pulmonary edema. We attempted to confirm whether pulmonary edema can be reproduced under intravital microscopy in a model of transfusion-related acute lung injury (TRALI) using MHC class I-specific antibody. Materials and Methods: The surface pulmonary microcirculation was observed using an epi-fluorescence microscope through a thoracic window in 50 male mice. Monoclonal MHC class I-specific antibody (Ab) was administered to the animals, while the control group received saline. The leukocytes and macro-molecular leakage in the pulmonary circulation were analyzed. Results: Leukocytes accumulated in the capillaries $(52.5 \pm 12.7$ leukocytes per designated area in Ab group vs. 20.8 \pm 3.1 in control). The air-containing alveolus area significantly shrank from 2,224.9 $\pm 934.9{\mu m^{2}}^{2}$ to $509.7 \pm 380.8 \mu^{2}$ in the Ab group. Conclusion: Pulmonary edema develops rapidly following leukocyte accumulation in the lung. We confirmed that leukocyte accumulation without an underlining condition is sufficient to induce pulmonary edema.
\end{abstract}

Leukocyte accumulation is a typical pathological alteration in acute pulmonary distress. The involvement of anti-HLA or anti-neutrophil antibody in the establishment of transfusionrelated acute lung injury (TRALI) has been widely accepted

This article is freely accessible online.

Correspondence to: Prof. Takehiko Iijima, Department of Perioperative Medicine, Division of Anesthesiology, School of Dentistry, Showa University, Kitasenzoku 2-1-1 Ohta City, Tokyo 145-8515, Japan. Tel: +81 0337871151, Fax: +81 0337870248, e-mail iijima@dent.showa-u.ac.jp

Key Words: Leukocyte, pulmonary edema, wet lung weight, MHC class I-specific antibody, intravital scope, microcirculation.
(1). This involvement has also been confirmed epidemiologically, since a male-only policy (not involving anti-HLA or anti-neutrophil antibody) effectively reduced the incidence of TRALI in a human cohort $(2,3)$. Since non-specific immunoglobulin reportedly reduces the severity of lung injury (4), the first step in this immunological response is undoubtedly the interaction between anti-HLA or antineutrophil antibody and cognate antigen. However, a single factor, i.e., anti-HLA or anti-neutrophil antibody, cannot necessarily reproduce TRALI, even if cognate antibody is administered (5). Instead, additional factors, such as sepsis, seem to be required to establish the pathological entity of pulmonary distress as TRALI. The detailed pathophysiology of TRALI remains to be elucidated. However, the basic scenario is thought to involve the accumulation of leukocytes and the subsequent hyperpermeability of alveolar vessels $(6,7)$.

Intravital microscopy (IVM) is a potential tool for describing the behavior of microcirculation, enabling us to clarify what happens in local areas under different physiological and pathophysiological conditions (8). This technique can be used to clarify the time-sequential establishment of pulmonary distress and the relationship between activated leukocytes and vascular hyperpermeability (7). The first and most representative model for TRALI is BALB/c mice treated with a monoclonal antibody to $\mathrm{H} 2 \mathrm{Kd}$, known as the MHC-class I antibody model (9). This model results in an increase in extravascular lung water, peripheral blood neutropenia, and the accumulation of neutrophils in the lung microvasculature. This model has been further investigated by several groups (10). Although these previous reports are informative regarding the pathophysiological aspects of TRALI, direct observations of the pathological development of TRALI caused by MHC class I-specific antibody have not yet been reported.

Herein, we developed a modified lung window model using a more convenient protocol for IVM of the murine lung microcirculation to further explore the role of leukocyte activation on the development of pulmonary edema. 


\section{Materials and Methods}

Animals and ethical statement. The experiments were performed using 10-to-12-week-old male BALB/c mice (Japan SLC, Inc., Shizuoka, Japan) weighing 24-28 $\mathrm{g}(\mathrm{n}=50)$. A lung window was surgically inserted in 10 mice for pulmonary microcirculation observations (control group, $\mathrm{n}=5$; Ab group (monoclonal antibodies against $\mathrm{H} 2 \mathrm{Kd}(\mathrm{mAb}-\mathrm{H} 2 \mathrm{Kd})$ administered group, described later), $\mathrm{n}=5$ ). An arterial blood gas analysis and a complete blood count were also performed (control group, $n=5$; Ab group, $n=5$ ).

We have made an independent experimental group to correctly measure the ex vivo wet and dry lung to avoid the influence of invasive procedure. Additional animals (control group, $\mathrm{n}=15$; $\mathrm{Ab}$ group, $n=15$ ) were subjected to septic condition and control condition. The ex vivo wet and dry lung masses were weighed. All the experimental protocols were approved by the Committee for Animal Experiments at the National Institute of Public Health (protocol number 26-002) and were performed in accordance with all the guidelines and laws for animal experiments in Japan.

Preparation of a lung window. Surgical preparation of the lung window was basically performed using the method of Tabuchi et al. (8), with some modifications. Each mouse was anesthetized with the inhalation of sevoflurane (Pfizer Inc., New York City, NY, USA) using a small animal anesthetizer (Model MK-A110D; Muromachi Kikai Co., Ltd., Tokyo, Japan). The body core temperature was kept constant throughout the experiment using a heating pad system for rodents (FHC-HPS; Mutomachi Kikai Co., Ltd.). A 20-gauge intravenous cannula (Surflo I.V. Catheter; Terumo Co., Tokyo, Japan) was inserted intratracheally and promptly connected to a respirator (MK-V100; Muromachi Kikai Co. Ltd.) to enable respiration. Throughout the experiment, the $\mathrm{FiO}_{2}$ was set at 0.50 and the breathing frequency was maintained at 100 breaths/min. This respirator works on a volume-dependent mode, therefore, peak inspiratory pressure cannot be set up. Under these conditions, an anesthetized state was maintained with $1.5 \%$ sevoflurane. The skin over the right rib cage was excised, and the underlying muscles of the region were dissected to expose the ribs and intercostal muscles of the right anterior-lateral thoracic wall. By performing a partial resection between the third and sixth ribs, an approximately $8-\mathrm{mm}$ circular window was excised in a manner such that the lower margin of the upper right lung lobe was exposed in the center of the window. A circular cover glass with a diameter of $7 \mathrm{~mm}$ (Matsunami Glass Ind., Ltd., Osaka, Japan) was tightly layered on a small piece of polyvinylidene membrane $(3 \mathrm{~cm} \times 3 \mathrm{~cm}$, New Kure-wrap, Kureha Co., Tokyo, Japan) and glued in place using a cyanoacrylates glue. This glass was then positioned so that the membrane side was in contact with the surface of the lung; the periphery of the window and the membrane were then tightly sealed with cyanoacrylates glue. The remaining air in the intrathoracic space was expelled using a syringe with a 30-gauge needle that was used to puncture the periphery of the window, causing the lung surface to come in contact with the transparent window membrane. The window was implanted (Figure $1 \mathrm{~A}$ and $\mathrm{B})$, and the mouse was then transferred to the stage of an intravital microscope while continuing body-heating and ventilation. In addition, the positive end-expiratory pressure (PEEP) was set at $10 \mathrm{mmH}_{2} \mathrm{O}$ throughout the observation period.

Real-time imaging of lung microcirculation using intravital microscopy. It needed approximately $30 \mathrm{~min}$ to prepare lung window, then the animal was mounted on an epi-fluorescence microscope (BX51WI; Olympus Co., Tokyo, Japan) equipped with a motorized stage, an EMCCD camera (Rolera MGI Plus; QImaging, Surrey, Canada), and a $20 \times$ long working distance objective lens (LUCPLFLN 20X, N.A.=0.45; Olympus Co.) for intravital microscopy. We determined time 0 when we administered antibody.

A series of fluorescence images were captured with an exposure time of $5 \mathrm{msec} /$ frame and a 20 -msec time-interval using a fluorescent filter for FITC and rhodamine. FITC-labeled dextran [average molecular weight, $70 \mathrm{kDa}$ (FITC-dex70); Sigma-Aldrich Co (St Louis, MO, USA)] and Rhodamine 6G (Wako Pure Chemicals Ind., Ltd., Osaka, Japan) were used to obtain images to detect vascular permeability and leukocyte behavior, respectively. A $100-\mu$ l bolus of FITC-dex70 (1\% in saline) and a $100-\mu$ l bolus of Rhodamine $6 \mathrm{G}(0.02 \%$ in saline) were injected into the caudal vein. To avoid movement artifacts caused by spontaneous respiratory motion, xylazine hydrochloride (10 mg/kg body weight; Wako Pure Chemicals Industries, Ltd.) was intraperitoneally injected prior to the image recording. In addition, the image recording was limited to a maximum of three times at an interval of at least 1 min per mouse to avoid artificial microcirculatory disorder.

Image analysis. Fluorescent images obtained using intravital microscopy were analyzed offline using Image J software (US National Institutes of Health, Bethesda, MD, USA). All the images were randomly renamed for blinded analysis and were analyzed by three independent observers to ensure the validity of the analysis.

For the quantitative analysis of leukocyte behavior, an image that was in focus was selected from the sequential image file of rhodamine $6 \mathrm{G}$, which were obtained at 0 time point and $30 \mathrm{~min}$ in each group. Each observer set three regions of interest (ROIs) with a square area $(150 \mu \mathrm{m} \times 150 \mu \mathrm{m})$ per image and counted the number of leukocytes inside the ROIs. The mean number of the three ROIs was regarded as the representative number for each mouse.

For the quantitative image analysis of FITC-dex70 images, we calculated the mean area of an alveolus, since alveoli are identifiable as black areas on FITC-dex70 images, which were obtained at 0 time point and at $30 \mathrm{~min}$ in each group. From the sequential image file for FITC-dextran, one image that was in focus was selected, and the area of each alveolar region was measured. Measurements were performed for at least 15 alveoli, and the mean alveolar area was obtained.

MHC I mAb production and administration. Monoclonal antibodies against $\mathrm{H} 2 \mathrm{~K}^{\mathrm{d}}\left(\mathrm{mAb}-\mathrm{H} 2 \mathrm{~K}^{\mathrm{d}}\right)$ were produced according to a method described by Looney et al. (9), with some modifications. A hybridoma (34-1-2S) that produces $\mathrm{mAb}-\mathrm{H} 2 \mathrm{~K}^{\mathrm{d}}$ was purchased from the American Type Culture Collection (Manassas, VA, USA). The isolated $\mathrm{mAb}-\mathrm{H} 2 \mathrm{Kd}$ was confirmed to produce a single band on gel electrophoresis and to bind specifically to neutrophils of BALB/c mice using flow cytometry (data not shown). The mice were given an i.v. volume-matched injection (100-150 $\mu$ l) of either $\mathrm{mAb}-\mathrm{H} 2 \mathrm{~K}^{\mathrm{d}}(4.5 \mathrm{mg} / \mathrm{kg}$ ) (Ab group) or saline via the caudal vein (control group).

Blood variables and blood gas analysis. Arterial blood samples were collected for analysis. A blood cell count was performed using a blood analyzer (VetScan HM2 Hematology System; Abaxis, Union 

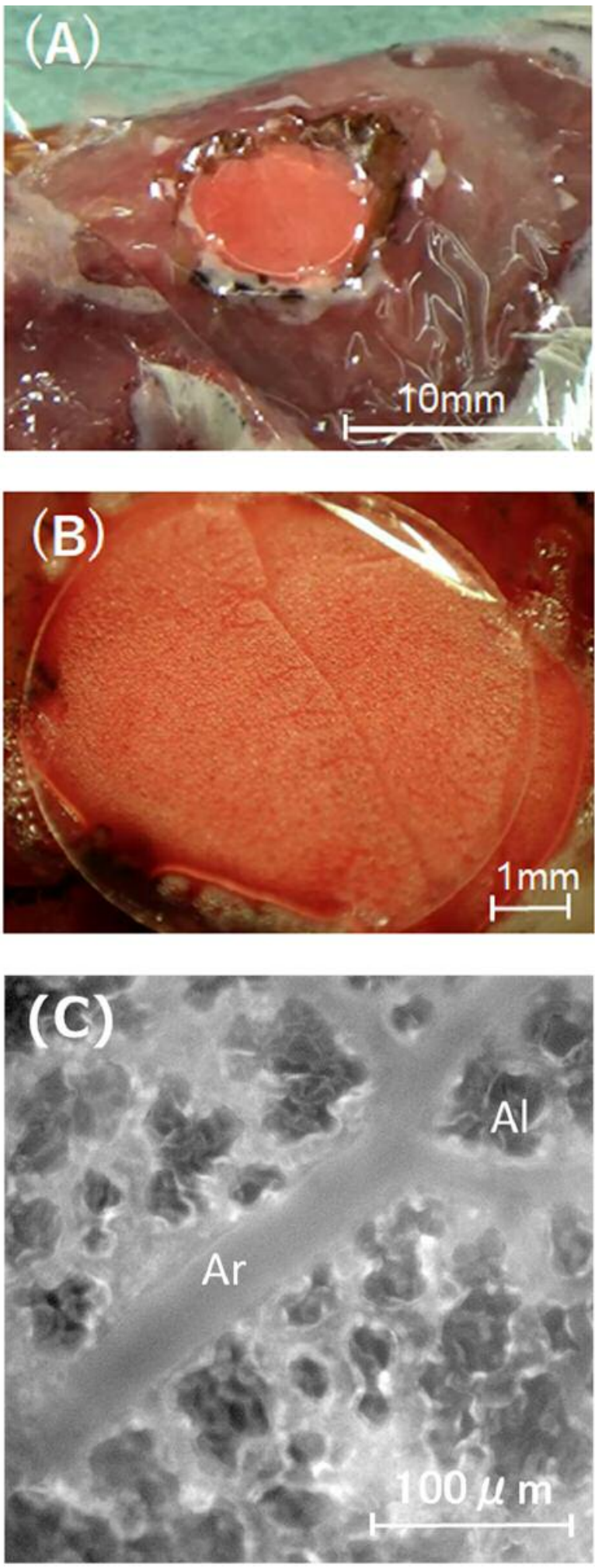

Figure 1. Lung window for intravital microscopy. Overview of the lung window $(A)$ and a magnified area $(B)$. The capillary and artery were demarcated by fluorescence using FITC-dex70 (C). The black follicular images represent the alveoli. The scale bars show $1 \mathrm{~cm}$ in (A), $1 \mathrm{~mm}$ in $(B)$, and $100 \mu \mathrm{m}$ in $(C)$, respectively. Ar: Artery; Al: alveolus.
City, CA, USA), and a blood gas analysis was performed to determine oxygen saturation $\left(\mathrm{sO}_{2}\right)$, arterial pressure of oxygen $\left(\mathrm{PaO}_{2}\right)$ and carbon dioxide $\left(\mathrm{PaCO}_{2}\right), \mathrm{pH}$, and bicarbonate $\left(\mathrm{HCO}_{3}{ }^{-}\right)$ level (i-STAT; Abbott Point of Care Inc., Princeton, NJ, USA).

Measurement of excess lung water mass. The chest of each mouse was opened under continuous ventilation at $30 \mathrm{~min}$ after $\mathrm{mAb}$ administration. The pulmonary arteriovenous vein and the main bronchus were ligated at the base of the left lung, and the lung tissue was excised immediately below the ligated part. The excised left lung was placed into a centrifuge tube to prevent it from drying, and the wet weight was measured. Thereafter, the lungs were dried using a vacuum-drying technique. The increase in the total lung water content was then assessed by calculating the wet:dry ratio. The wet:dry ratio was calculated as the wet weight divided by the dry weight. The lung water mass index (LWI) was calculated using the following formula: LWI=wet weight $(\mathrm{mg})$ /body weight $(\mathrm{g})$.

Statistical analysis. The measured data are presented as the mean \pm SD. The number of mice used in each experiment is described in the figure legends. The statistical analyses were performed using SPSS Statistics software (Version 22; Japan IBM Co., Tokyo, Japan). Comparisons were made using an unpaired Student $t$-test, and a $p$-value of $<0.05$ was considered significant.

\section{Results}

Intravital microscopic images. Patent alveoli were confirmed as multi-follicular black fields under an intravital microscope and were surrounded by alveolar tissue, including capillaries (Figure 1C). A clear angiogram was observed in the control group, suggesting the maintenance of the pulmonary arterioles and complex capillary network.

We have confirmed that leukocytes began to roll on the vascular wall and accumulate immediately after $\mathrm{Ab}$ administration, while leukocyte rolling and accumulation have not been confirmed during mechanical ventilation before $\mathrm{Ab}$ administration.

Occasionally, the blood flow was stopped by leukocyte aggregation. The images obtained in the $\mathrm{Ab}$ group showed leukocyte accumulation in the alveolar vessels (Figure 2B), unlike the images obtained in control mice (Figure 2A). Leukocyte aggregation increased significantly in the $\mathrm{Ab}$ group, while the number of the aggregated leukocytes remained unchanged in the control group (Figure 2C). The accumulation of leukocytes in the pulmonary microcirculation was accompanied by a decrease in leukocytes and platelets in large veins (Table I).

Interstitial lung edema was evaluated using fluorescent microscopy with FITC-dex70. Alveolar patency was maintained in the control group (Figure 3A), while the fluorescent interstitium became predominant in images obtained in the $\mathrm{Ab}$ group (Figure $3 \mathrm{~B}$ ). The patent area decreased significantly in the $\mathrm{Ab}$ group, as did the mean alveolar area $(p<0.01)$, while the patency remained unchanged in the control group (Figure 3C). 

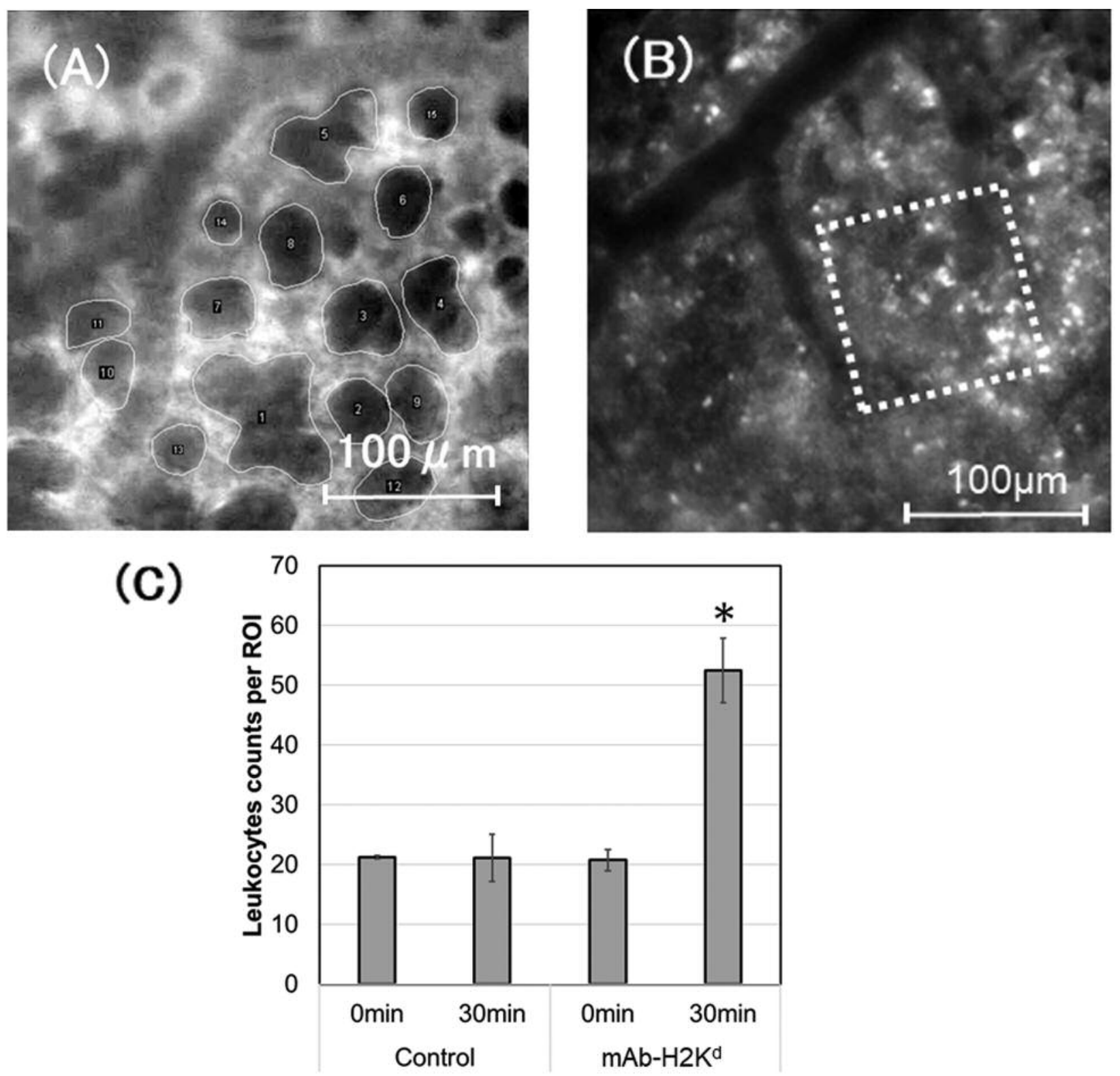

Figure 2. Fluorescence intravital microscopic images of mouse lung using Rhodamine $6 G$ infusion and quantitative image analysis of leukocyte accumulation $(A, B)$. Representative microscopic images of leukocytes at 30 min after the administration of saline $(A)$ and $m A b-H 2 K d(B)$. The whitedotted boxes in these images indicate the regions of interest (ROIs, $150 \mu \mathrm{m} \times 150 \mu \mathrm{m})$ used for the quantitative image analysis of leukocyte accumulation. (C) Comparison of leukocyte counts per ROI in alveoli. The number of leukocytes that had accumulated inside the alveolar microvasculature was counted in a blinded manner. The leukocyte count significantly increased from $20.8 \pm 3.1$ to $52.5 \pm 12.7$ in the Ab group after $m A b-H 2 K d$ administration, while the leukocyte count remained unchanged in the control group from $21.2 \pm 0.5$ at 0 time point to $21.1 \pm 7.7$ at 30 min. The scale bar in each figure represents $100 \mu \mathrm{m} .{ }^{*} p<0.05 \mathrm{vs}$. control.

Respiratory conditions after Ab administration. We did not confirm deoxygenation even after $\mathrm{Ab}$ administration, as confirmed by the blood gas analysis (Table II). The average $\mathrm{P} / \mathrm{F}$ ratios were $287 \pm 97$ for the $\mathrm{Ab}$ group and $418 \pm 132$ for the control group $(p>0.05)$. No other remarkable changes were observed even after Ab administration. Only the peripheral leukocyte count decreased significantly (Table I).

Evaluation of lung edema. The wet lung weight was higher in the Ab group, resulting in a significantly higher wet:dry ratio in the $\mathrm{Ab}$ group, compared with the control group 

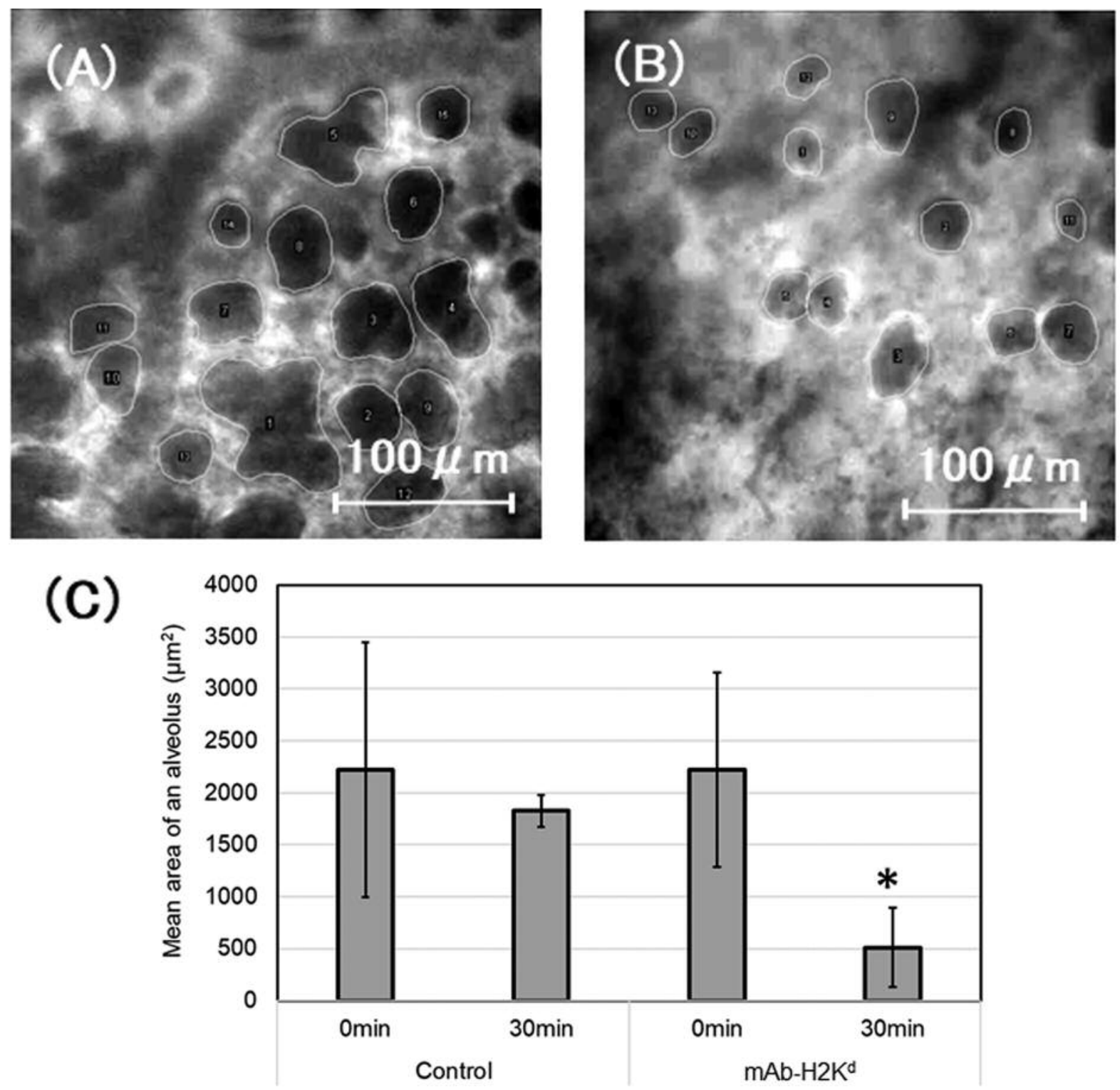

Figure 3. Fluorescence microscopic images of lung microvasculature using FITC-dex70 and quantitative image analysis. IVM images of the lung microvasculature obtained $30 \mathrm{~min}$ after the administration of saline (A) or $\mathrm{mAb}-\mathrm{H} 2 \mathrm{~K}^{d}(\mathrm{~B})$. Each white-lined circle in the images indicates an alveolus. (C) Comparison of mean alveolar area between control and $m A b-H 2 K^{d}$-administered mice. Each alveolar area was measured in a blinded

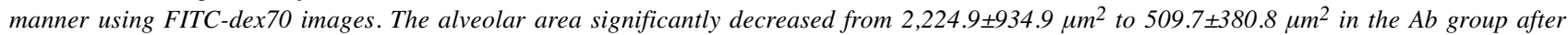
$m A b-H 2 K d$ administration, while the alveolar area remained unchanged in the control group from 2,218.1 $\pm 1225.7 \mu m^{2}$ at 0 time point to $1825.3 \pm 155.6 \mu \mathrm{m}^{2}$ at $30 \mathrm{~min}$. This finding suggests that the penetration of water into the interstitium was caused by the enhancement of vascular permeability, resulting in a widened interstitial area. The scale bar in each figure represents $100 \mu \mathrm{m} . * p<0.05 \mathrm{vs}$. control.

$(6.80 \pm 3.88$ in the $\mathrm{Ab}$ group vs. $4.64 \pm 1.16$ in the control group) $(p<0.05)$ (Figure 4).

\section{Discussion}

The present study demonstrated that pulmonary edema can be induced by the aseptic activation of leukocytes. We demonstrated leukocyte accumulation in the pulmonary tissue and adherence of these leukocytes to the pulmonary capillaries, followed by the development of pulmonary edema. Regarding the establishment of the TRALI pathology, TRALI has been hypothesized to follow either a 'two hit model' (11) or a 'threshold model' $(12,13)$. MHCantibodies are considered to represent a 'second hit'. Our 
Table I. Blood count before and at 30 min after $m A b-H 2 K^{d}$ administration.

\begin{tabular}{lcc}
\hline & Control (Saline) & $\mathrm{mAb}-\mathrm{H} 2 \mathrm{~K}^{\mathrm{d}}$ \\
\hline WBC $\left(\times 10^{-9} / 1\right)$ & $6.15 \pm 1.05$ & $4.69 \pm 2.48^{*}$ \\
$\mathrm{RBC}\left(\times 10^{-9} / 1\right)$ & $9.83 \pm 0.29$ & $11.27 \pm 1.09$ \\
$\mathrm{Hb}(\mathrm{g} / \mathrm{dl})$ & $15.65 \pm 0.48$ & $17.11 \pm 1.39$ \\
$\mathrm{Hct}(\%)$ & $48.78 \pm 1.35$ & $53.43 \pm 4.82$ \\
PLT $\left(\times 10^{-9} / 1\right)$ & $308.50 \pm 193.18$ & $128.42 \pm 87.85$ \\
\hline
\end{tabular}

WBC: White blood cells; RBC: red blood cells; Hb: hemoglobin; Hct: hematocrit; PLT: platelets. ${ }^{*} p<0.05 v s$. control.

Table II. Arterial blood gas parameters before and at $30 \mathrm{~min}$ after $\mathrm{mAb}$ $H 2 K^{d}$ administration .

\begin{tabular}{lcc}
\hline & Control (saline) & $\mathrm{mAb}-\mathrm{H} 2 \mathrm{~K}^{\mathrm{d}}$ \\
\hline $\mathrm{FiO}_{2}$ & 0.5 & 0.5 \\
$\mathrm{pH}$ & $7.30 \pm 0.07$ & $7.26 \pm 0.01$ \\
$\mathrm{PaCO}_{2}(\mathrm{mmHg})$ & $28.8 \pm 4.4$ & $34.5 \pm 6.7$ \\
$\mathrm{PaO}_{2}(\mathrm{mmHg})$ & $143.3 \pm 48.7$ & $209.0 \pm 66.1$ \\
$\mathrm{BEecf}^{(\mathrm{mmol} / \mathrm{l})}$ & $-4.3 \pm 12.2$ & $-11.4 \pm 3.6$ \\
$\mathrm{HCO}_{3}{ }^{-}(\mathrm{mmol} / \mathrm{l})$ & $14.0 \pm 1.9$ & $15.7 \pm 3.4$ \\
$\mathrm{TCO}_{2}(\mathrm{mmol} / \mathrm{l})$ & $14.8 \pm 2.0$ & $16.6 \pm 3.6$ \\
$\mathrm{SaO}_{2}(\%)$ & $97.8 \pm 2.0$ & $99.4 \pm 0.8$ \\
$\mathrm{Lac}^{(\mathrm{mmol} / \mathrm{l})}$ & $4.0 \pm 1.1$ & $2.7 \pm 0.7$ \\
$\mathrm{P} / \mathrm{F} \mathrm{ratio}(\mathrm{mmHg})$ & $287 \pm 97$ & $418 \pm 132$ \\
\hline
\end{tabular}

$\mathrm{FiO}_{2}$ : Fraction of inspired oxygen; $\mathrm{PaCO}_{2}$ : partial pressure of carbon dioxide in arterial blood; $\mathrm{PaO}_{2}$ : partial pressure of oxide in arterial blood; BEecf: Base excess; $\mathrm{HCO}_{3}{ }^{-}$: Bicarbonate; $\mathrm{TCO}_{2}$ : total carbon dioxide; $\mathrm{SaO}_{2}$ : arterial blood oxygen saturation. $\mathrm{P} / \mathrm{F}$ ratio calculated value from $\mathrm{PaO}_{2} / \mathrm{FiO}_{2}$. No statistical significance was confirmed in any parameter $(p<0.05)$

model did not include septic conditions. Thus, leukocyte accumulation in the pulmonary tissue may not require a "first hit." In the 'threshold model', the threshold is formed by the level of priming of lung leukocytes, mainly neutrophils, and the ability of mediators in the transfusion product to activate these primed neutrophils (14). Since we observed that MHC class I-specific antibody per se induces a sequential pathophysiological process, our results may support the threshold theory.

The first animal TRALI model using the passive transfusion of an MHC class I mAb $(\mathrm{H} 2 \mathrm{Kd})$ to mice was reported by Looney et al. (9). They demonstrated that the injection of this mAb rapidly induced pulmonary vascular leakage, thereby increasing the lung water content, and was associated with temporary neutropenia. This model has been subsequently used by several research groups (10) and has been thoroughly reviewed $(11,15)$. Several hypothetic mechanisms for the animal TRALI model have been proposed. Anti-MHC-class I (H-2K) monoclonal Ab (mAb)

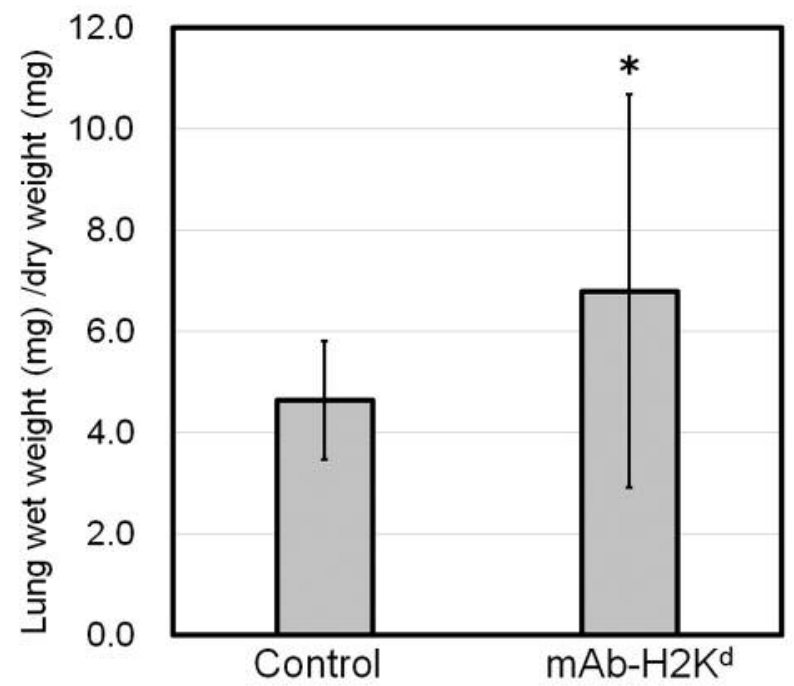

Figure 4. Lung wet weight index (LWI) of excised lungs. Thirty min after $m A b-H 2 K^{d}$ (or saline) administration, the left lung was excised and weighed and the LWI was calculated. The LWI in the $m A b-H 2 K^{d}$ administered mice $(3.04 \pm 0.67$, mean $\pm S D, n=13)$ was higher than that in the control mice $(2.39 \pm 0.26, n=13) . * p<0.05$ vs. control.

has been reported to activate complement from nonhematopoietic cells, which could explain the initiation of TRALI (16). Another study showed that deposition of activated neutrophils and platelets, which are primed by LPS, is required to induce TRALI (17). Thus, several complicated steps, including complement activation from endothelial cell, neutrophil priming and leukocyte binding with selection (10) are required to develop TRALI. H-2K monoclonal antibody acts on endothelial cells (non-hematopoietic) and/or leukocytes (hematopoietic) to initiate lung edema.

Our direct observation of water retention in the alveoli is the first report in a TRALI model. This direct confirmation of pulmonary edema in the lung window provided us with information regarding how quickly pulmonary edema develops immediately after leukocyte accumulation. The focus of investigation of the TRALI mechanism should now move to analyze the sequential steps of the molecular immunological mechanism leading to vascular hyperpermeability. Real-time observations in regions of interest could help to elucidate the pathological sequence of the establishment of lung injury following the initiation of leukocyte activation. In this study, we presented an image at one time point. Time-sequential images would help analyze the mechanism, however, photodamage on the lung precluded the repeated acquisition of the images. Preventing photodamage should be the subject to overcome in further studies.

We did not confirm significant desaturation in the Ab group, although severe water retention was observed. Pulmonary 
vessels have a property, so-called, hypoxic pulmonary vasoconstriction. As long as patent alveolus remains in the lung, oxygenation could be maintained above desaturation level. Even in swine model, we rarely confirm severe desaturation even after anti-MHC-I specific antibody (5).

We confirmed the presence of pulmonary edema by means of direct alveoli imaging and quantified the associated water retention in the alveolar tissue. The lung wet-dry ratio in the $\mathrm{Ab}$ group in our study was comparable with that of an ARDS model in other studies $(18,19)$.

In this study, we obtained images of the lung microcirculation using intravital microscopy. IVM of the murine lung requires several methodological challenges to be overcome, although similar visual access to the lung has been previously created in larger animals (18). In mice, this approach is hampered by the proportions of the small murine chest wall vs. the relatively large access area required for IVM. To counteract motion artifacts, a negative pressure is typically applied via suction manifolds and a series of bore holes (20, 21). Razavi et al. (22) and Looney et al. (15) were able to overcome these difficulties in their reports on the preparation of lung windows in mice, although their methods require special equipment. Following these studies, Tabuchi et al. (8) reported an original model for IVM of the pulmonary microcirculation involving controlled ventilation. Our method was based on Tabuchi et al.'s study with some modifications for added convenience and ease of preparation. The beneficial features of our model are as follows: 1) mechanical control to maintain the intrathoracic pressure via a catheter is not required, and 2) a flat window is created using a circular glass.

Several limitations of this study should be considered. In terms of observation periods, since the lung window model developed in this study can be used for periods no longer than $30 \mathrm{~min}$, subsequent time-dependent changes should be observed in future studies. The extravasation of water in the alveolus has a strong impact, suggesting pulmonary distress. However, the time course of the pulmonary edema and the subsequent desaturation should have been confirmed for a longer time period. A chronic model enabling imaging conditions to be sustained for longer periods could expand future experimental periods.

In conclusion, we confirmed that interstitial expansion arising from hyperpermeability and the accumulation of leukocytes in the alveolar microenvironment was caused by MHC class I-specific antibody without an underlining specific condition. IVM using a lung window might contribute to the further clarification of the pathogenetic mechanisms of immunological lung injury.

\section{Conflicts of Interest}

The Authors have no conflicts of interest to declare regarding this study.

\section{Authors' Contributions}

$\mathrm{HO}$ and AU designed and performed the experiments, analyzed the data, and wrote the paper; TI developed the experimental concept, analyzed the data, and wrote the paper.

\section{Acknowledgements}

This work was supported by the Japan Society of the Promotion of Science (JSPS) KAKENIHI, Grant-in-Aid for Scientific Research (C) $16 \mathrm{~K} 11762$ to TI.

The Authors would like to thank Ms. Myrna Harrod for her English language review. The Authors would also like to thank Mr. Masaya Aoki, Mr. Keita Umino, Ms. Machiko Maeda, Ms. Masako Osawa, Ms. Kasumi Yamanaka and Ms. Miyuu Nobumasa for their assistance with the experiments, and Dr. Kohji Uzawa, Dr. Shin Ohtani, and Dr. Hanae Kataoka for their expert advice.

\section{References}

1 Bux J, Becker F, Seeger W, Kilpatrick D, Chapman J and Waters A: Transfusion-related acute lung injury due to hla-a2-specific antibodies in recipient and nb1-specific antibodies in donor blood. Br J Haematol 93(3): 707-713, 1996. PMDI: 8652399.

2 Nakazawa H, Ohnishi H, Okazaki H, Hashimoto S, Hotta H, Watanabe T, Ohkawa R, Yatomi Y, Nakajima K, Iwao Y, Takamoto S, Shimizu M and Iijima T: Impact of fresh-frozen plasma from male-only donors versus mixed-sex donors on postoperative respiratory function in surgical patients: A prospective case-controlled study. Transfusion 49(11): 2434-2441, 2009. PMID: 19624605. DOI: 10.1111/j.1537-2995.2009. 02321.x

3 Chasse M, McIntyre L, English SW, Tinmouth A, Knoll G, Wolfe D, Wilson K, Shehata N, Forster A, van Walraven C and Fergusson DA: Effect of blood donor characteristics on transfusion outcomes: A systematic review and meta-analysis. Trans Medicine Rev 30: 69-80, 2016. PMID: 26920039. DOI: 10.1016/j.tmrv.2016.01.002

4 Semple JW, Kim M, Hou J, McVey M, Lee YJ, Tabuchi A, Kuebler WM, Chai $\mathrm{ZW}$ and Lazarus AH: Intravenous immunoglobulin prevents murine antibody-mediated acute lung injury at the level of neutrophil reactive oxygen species (ROS) production. PLoS One 7(2): e31357, 2012. PMID: 22363629. DOI: $10.1371 /$ journal.pone.0031357

5 Okazaki H, Ishikawa O, Iijima T, Kohira T, Teranishi M, Kawasaki S, Saito A, Mikami Y, Sugiura A, Hashimoto S, Shimada E, Uchikawa M, Matsuhashi M, Tsuno NH, Tanaka M, Kiyokawa N, Fujimoto J, Nagase T, Tadokoro K and Takahashi $\mathrm{K}$ : Novel swine model of transfusion-related acute lung injury. Transfusion 54(12): 3097-3107, 2014. PMID: 24965098. DOI: 10.1111/trf.12766

6 Morsing KSH, Peters AL, van Buul JD and Vlaar APJ: The role of endothelium in the onset of antibody-mediated trali. Blood Rev 32: 1-7, 2017. PMID: 28823763. DOI: 10.1016/j.blre.2017.08.003

7 Seeger W, Schneider U, Kreusler B, von Witzleben E, Walmrath $\mathrm{D}$, Grimminger $\mathrm{F}$ and Neppert J: Reproduction of transfusionrelated acute lung injury in an ex vivo lung model. Blood 76(7): 1438-1444, 1990. PMID: 2207319.

8 Tabuchi A, Mertens M, Kuppe H, Pries AR and Kuebler WM: Intravital microscopy of the murine pulmonary microcirculation. 
J Appl Physiol (1985) 104(2): 338-346, 2008. PMID: 18006870 DOI: $10.1152 /$ japplphysiol.00348.2007

9 Looney MR, Su X, Van Ziffle JA, Lowell CA and Matthay MA: Neutrophils and their fc gamma receptors are essential in a mouse model of transfusion-related acute lung injury. J Clin Invest 116(6): 1615-1623, 2006. PMID: 16710475. DOI: $10.1172 / \mathrm{JCI} 27238$

10 Hidalgo A, Chang J, Jang JE, Peired AJ, Chiang EY and Frenette PS: Heterotypic interactions enabled by polarized neutrophil microdomains mediate thromboinflammatory injury. Nat Med 15(4): 384-391, 2009. PMID: 19305412. DOI: 10.1038/nm.1939

11 Peters AL, Van Stein D and Vlaar AP: Antibody-mediated transfusion-related acute lung injury; from discovery to prevention. Br J Haematol 170(5): 597-614, 2015. PMID: 25921271. DOI: 10.1111/bjh.13459

12 Sachs UJ, Hattar K, Weissmann N, Bohle RM, Weiss T, Sibelius $\mathrm{U}$ and Bux J: Antibody-induced neutrophil activation as a trigger for transfusion-related acute lung injury in an ex vivo rat lung model. Blood 107(3): 1217-1219, 2006. PMID: 16210340. DOI: 10.1182/blood-2005-04-1744

13 Sachs UJ: A threshold model for the susceptibility to transfusionrelated acute lung injury. Transfus Clin Biol 19: 109-116, 2012. PMID: 22677431. DOI: 10.1016/j.tracli.2012.03.003

14 Bux J and Sachs UJ: The pathogenesis of transfusion-related acute lung injury (trali). Br J Haematol 136(6): 788-799, 2007. PMID: 17341264. DOI: 10.1111/j.1365-2141.2007.06492.x.

15 Gilliss BM and Looney MR: Experimental models of transfusion-related acute lung injury. Transfus Med Rev 25: 111, 2011. PMID: 21134622. DOI: 10.1016/j.tmrv.2010.08.002

16 Strait RT, Hicks W, Barasa N, Mahler A, Khodoun M, Kohl J, Stringer K, Witte D, Van Rooijen N, Susskind BM and Finkelman FD: MHC class i-specific antibody binding to nonhematopoietic cells drives complement activation to induce transfusion-related acute lung injury in mice. J Exp Med 208(12): 2525-2544, 2011. PMID: 22025304. DOI: 10.1084/ jem.20110159
17 Looney MR, Nguyen JX, Hu Y, Van Ziffle JA, Lowell CA and Matthay MA: Platelet depletion and aspirin treatment protect mice in a two-event model of transfusion-related acute lung injury. J Clin Invest 119(11): 3450-3461, 2009. PMID: 19809 160. DOI: $10.1172 / \mathrm{JCI} 38432$

18 Zhang S, Wang P, Zhao P, Wang D, Zhang Y, Wang J, Chen L, Guo W, Gao H and Jiao Y: Pretreatment of ferulic acid attenuates inflammation and oxidative stress in a rat model of lipopolysaccharide-induced acute respiratory distress syndrome. Int J Immunopathol Pharmacol 32: 394632017750518, 2018. PMID: 29350567. DOI: 10.1177/0394632017750518

19 Voelker MT, Fichtner F, Kasper M, Kamprad M, Sack U, Kaisers UX and Laudi S: Characterization of a double-hit murine model of acute respiratory distress syndrome. Clin Exp Pharmacol Physiol 41(10): 844-853, 2014. PMID: 25115497. DOI: 10.1111/ 1440-1681.12283

20 Wagner WW, Jr.: Pulmonary microcirculatory observations in vivo under physiological conditions. J Appl Physiol 26(3): 375377, 1969. PMID: 5773180. DOI: 10.1152/jappl.1969.26.3.375

21 Kuhnle GE, Leipfinger FH and Goetz AE: Measurement of microhemodynamics in the ventilated rabbit lung by intravital fluorescence microscopy. J Appl Physiol (1985) 74(3): 1462-1471, 1993. PMID: 8482691. DOI: 10.1152/jappl.1993. 74.3.1462

22 Razavi HM, Wang le F, Weicker S, Rohan M, Law C, McCormack DG and Mehta S: Pulmonary neutrophil infiltration in murine sepsis: Role of inducible nitric oxide synthase. Am J Respir Crit Care Med 170(3): 227-233, 2004. PMID: 15059787. DOI: $10.1164 / \mathrm{rccm} .200306-846 \mathrm{OC}$
Received July 2, 2019

Revised July 17, 2019

Accepted July 18, 2019 